\title{
Is Alpha-Glucosidase Inhibition a Mechanism of the Antidiabetic Action of Garlic (Allium sativum)?
}

\author{
Patience Obih ${ }^{1}$, John-Clifford Obih ${ }^{2}$, Okai Arome ${ }^{1}$ \\ ${ }^{1}$ College of Pharmacy, Xavier University of Louisiana, New Orleans, LA, USA \\ ${ }^{2}$ Southern University at New Orleans, New Orleans, LA, USA \\ Email: *poobih@xula.edu
}

How to cite this paper: Obih, P., Obih, J.-C. and Arome, O. (2019) Is Alpha-Glucosidase Inhibition a Mechanism of the Antidiabetic Action of Garlic (Allium sativum)? Journal of Biosciences and Medicines, 7, 42-49.

https://doi.org/10.4236/jbm.2019.710004

Received: September 7, 2019

Accepted: October 12, 2019

Published: October 15, 2019

Copyright $\odot 2019$ by author(s) and Scientific Research Publishing Inc. This work is licensed under the Creative Commons Attribution International License (CC BY 4.0).

http://creativecommons.org/licenses/by/4.0/

\begin{abstract}
Objective: There has been a global surge in the number of diabetic cases. Many of the agents used as antidiabetic are either expensive or have side effects. Researchers are now turning their attention to phytotherapy as a viable alternative in the treatment of hyperglycemia. The aim of this study was to examine the inhibition of $\alpha$-glucosidase as a possible mechanism of antidiabetic action of garlic. Method: The inhibitory effect of different concentrations of garlic was examined for alpha-glucosidase inhibitory activity in a 96-well micro plate. Saccharomyces cerevisiae was used as the source of alpha-glucosidase and the assay was analyzed with a Thermo Scientific ${ }^{\oplus}$ Multiskan Spectropho-meter at an absorbance of $400 \mathrm{~nm}$. Result: The extracts of garlic exhibited a dose-dependent inhibition of alpha-glucosidase in comparison to acarbose. The IC50 of acarbose was $3.19 \pm 0.42 \mathrm{mg} / \mathrm{ml}$, for garlic, the IC50 was $16.93 \mathrm{mg} / \mathrm{ml}$. Conclusion: In this study, garlic oil showed some promise as an antidiabetic agent with a mechanism of action similar to acarbose and miglitol that are currently used as antidiabetics. It is hoped that carrying out further research on garlic will elucidate other mechanisms of action.
\end{abstract}

\section{Keywords}

Diabetes, Alpha-Glucosidase, Medicinal Plants, Garlic (Allium sativum), Mechanism of Action

\section{Introduction}

Diabetes is a chronic metabolic disease characterized by hyperglycemia due to decreased insulin secretion from pancreas, decreased insulin sensitivity, primarily in muscles cells or both. According to World Health Organization, 422 mil- 
lion people are diagnosed with diabetes worldwide [1]. From the National Diabetes statistics report, more than 100 million U.S. adults are now living with diabetes [2] [3]. Several drugs have been introduced to the market to treat diabetes. These include Insulin, other injectable and oral hypoglycemic medications. Examples of antidiabetic agents already in the market include sulfonylureas (glyburide), meglitinide analogs (repaglinide), D-Phenylalanine derivative (nateglinide), biguanide (metformin), thiazolidinediones (pioglitazone), alpha-glucosidase inhibitors (acarbose), (glucagone-like peptide-1 receptor agonist (exenatide), dipeptidy peptidase 4 (DPP-4) inhibitors (sitapliptin), and sodium-glucose co-transporter 2 (SGLT) inhibitor (canagliflozin) [4]. Despite all these drugs, diabetes continues to increase at an alarming rate. Despite the progress made with synthetic drugs, the search continues for newer drugs because the existing ones have limitations. Many of these drugs are expensive, produce many side effects and may precipitate noncompliance by the patients. Researchers are intensifying their efforts in drug discovery especially in natural products. Several medicinal plants such as curcumin have been shown to be effective in different stages of diabetes. It has been proposed to be used as one of the interventions in pre-diabetes therapy to prevent the progression of type 2 diabetes mellitus, (T2DM) due to its proven benefits and safety profile [5]. Plants are available and offer a wide field to search for natural effective oral hypoglycemic agents that have few or no side effects. More than 1200 plant species have been recorded to be used worldwide for their alleged hypoglycemic activity [6]. Acarbose, miglitol and viglibose have been introduced as alpha-amylase and alpha-glucosidase inhibitors and acarbose is derived from plant. The inhibition of intestinal $\alpha$-glucosidases delays the digestion and absorption of carbohydrates and consequently suppresses postprandial hyperglycemia [7] [8]. Some researchers now focus their studies on alpha-glucosidase inhibitors from plant origin. For instance, a study in Mexico screened the inhibitory effect of hydroethanolic extracts from 23 medicinal plants used in Mexico as antidiabetics on glucosidase obtained from rat intestinal mucosa [9]. Other workers also searched for plants that are more potent and with less side effect that can inhibit alpha-glucosidase [10] [11]. Inhibition of $\alpha$-amylase by phytotherapy is a possible method for treating T2DM. Amylase inhibitors also slow the liberation of glucose from carbohydrates, delaying its intestinal absorption and consequently postprandial hyperglycemia [12]. The possible use of alpha-amylase inhibitors from medicinal plant such as blueberry leaves, tamarind, lemon balm, rosemary, white kidney beans (hulls), and green tea as antidiabetics was demonstrated [13]. Two herbal products, Urtica dioica, (Urticaceae) and Juglans regia L. (Juglandaceae), were also tested for their alpha-amylase inhibitory activity. Both were found to be active inhibitors of amylase [14].

Antidiabetic agents produce their actions by various mechanisms. Apart from inhibition of amylase and alpha-glucosidase, other mechanisms exist. Examples are: 1) Effects on glucose uptake and glucose transporters; 2) Enhancers of insulin secretion and pancreatic $\beta$ cell proliferation; and 3) Inhibition of protein ty- 
rosine phosphatase 1B activity [11]. Due to the rise in incidence and the financial burden associated with diabetes, it is important to search for pharmacologic and natural compounds that are cost-effective and able to reduce glycemic level in patients. So far, there is no antidiabetic drug in the market that has no side effects. Prominent adverse effects of $\alpha$-glucosidase inhibitors like acarbose include flatulence, diarrhea, and abdominal pain. For these reasons, various traditional plants like Garlic (Allium savitum) have become popular in the world owing to their safer therapeutic benefits. Garlic is well known to contain an array of bioactive extracts that can be beneficial in reducing the risk of cardiovascular diseases, possess antitumor and antimicrobial activities [15]. The beneficial effects of garlic are mainly attributed to the presence of volatile sulfur compounds like alliin, allicin, diallyl disulfide, diallyl trisulfide, diallyl sulfide, S-allyl cysteine, ajoene and allyl mercaptan [15]. These garlic extracts have been shown to be effective in reducing insulin resistance [16]. Despite the wide use of garlic, not much is known about its mechanism of action in reducing hyperglycemia. Garlic is an excellent plant and needs to be researched more. The aim of this study was to examine if garlic produces its antidiabetic activity by inhibiting alpha-glucosidase. We proposed that inhibition of alpha-glucosidase is the mechanism by which garlic produces its antidiabetic effect.

\section{Materials and Methods}

\subsection{Materials}

Garlic oil was purchased from Sigma Aldrich USA, Alpha-glucosidase Saccharomyces cerevisiae (Sigma-Aldrich USA), 4-Para-nitrophenyl- $\alpha$-D-glucopyranoside (PNPG) and acarbose, were purchased from Sigma-Aldrich, 0.2 buffer pH 6.8 was purchased from Alfa Aesar (USA).

\subsection{Garlic Extract}

Garlic extract (diallyl trisulfide) from Sigma-Aldrich was prepared by dissolving $100 \mathrm{mg}$ of garlic oil in a mixture of DMSO and $0.2 \mathrm{M}$ buffer $\mathrm{pH}$ 6.8. to make a concentration of $25 \mathrm{mg} / \mathrm{ml}$. The experiment was carried out in assays prepared in 96 micro-well plates. The absorbance was measured on Thermo Scientific Multiskan spectrophotometer at $400 \mathrm{~nm}$.

\subsection{The Alpha-Glucosidase Protocol}

Alpha-Glucosidase inhibition assay was done according to the method of Remirez et al. [17] with modification. A total of $2.7 \mathrm{mg}$ of Saccharomyces cerevisiae (Sigma-Aldrich USA) was dissolved in $2.7 \mathrm{~mL}$ of $0.2 \mathrm{M}$ Phosphate buffer. From this solution, $1 \mathrm{~mL}$ was added to $200 \mathrm{mg}$ of Bovine Serum Albumin (BSA) in $99 \mathrm{~mL}$ of $0.2 \mathrm{M}$ Phosphate buffer. Each test well consisted of $2.5 \mu \mathrm{L}$ of various dilutions of garlic extract, $122.5 \mu \mathrm{l}$ of $0.2 \mathrm{M}$ phosphate buffer and $62.5 \mu \mathrm{L}$ of 5 $\mathrm{mM}$ para-nitrophenyl- $\alpha$-D-glucopyranoside (PNPG) USA. This was incubated at $37^{\circ} \mathrm{C}$ for $5 \mathrm{~min}$ before adding alpha-glucosidase. After incubating for $5 \mathrm{mi}$ nutes, $62.5 \mu \mathrm{L}$ of $\alpha$-glucosidase solution was added to the mixture. The mixture 
was incubated again for 5 mins. Alpha-glucosidase activity was determined on a Thermo Scientific Multiscan Spectrophotometer at an absorbance of $400 \mathrm{~nm}$. Acarbose was used as a positive control of $\alpha$-glucosidase inhibition and was measured similarly. The alpha-glucosidase inhibitory activity was expressed as percentage inhibition. The experiments were perfomed in triplicate.

$$
\% \text { Inhibition }=\frac{\text { A } 410 \text { control }- \text { A } 410 \text { test }}{\text { A } 410 \text { control }} * 100
$$

The IC50 values of garlic (inhibitor concentration at which 50\% inhibition of the enzyme activity occurs) was determined by performing the assay as above with varying concentrations of the garlic extracts ranging from 20 to $100 \mathrm{mg} / \mathrm{ml}$. The IC50 values were determined from plots of percent inhibition versus log inhibitor concentration and calculated by non-linear regression analysis from the mean inhibitory values.

\section{Results}

Figure 1 shows garlic bulbs. The results of this study are shown in Figure 2 and Figure 3. The graphs show a dose-dependent inhibition of alpha-glucosidase by acarbose and garlic using Saccharomyces cerevisiae as the source of alpha-glucosidase. The results are expressed as mean \pm standard deviation. The IC50 was $2.19 \mathrm{mg} / \mathrm{ml}$ for acarbose and $16.93 \mathrm{mg} / \mathrm{ml}$ for garlic oil. In comparison, acarbose produced a greater inhibition of alpha-glucosidase than garlic. The active ingredient in garlic oil from sigma is Diallyl trisulfide. The refined form of acarbose was used in this study.

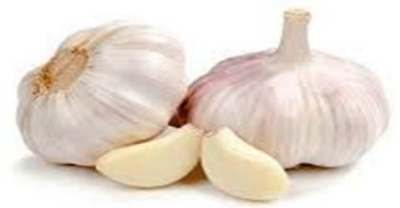

Figure 1. Garlic bulbs.

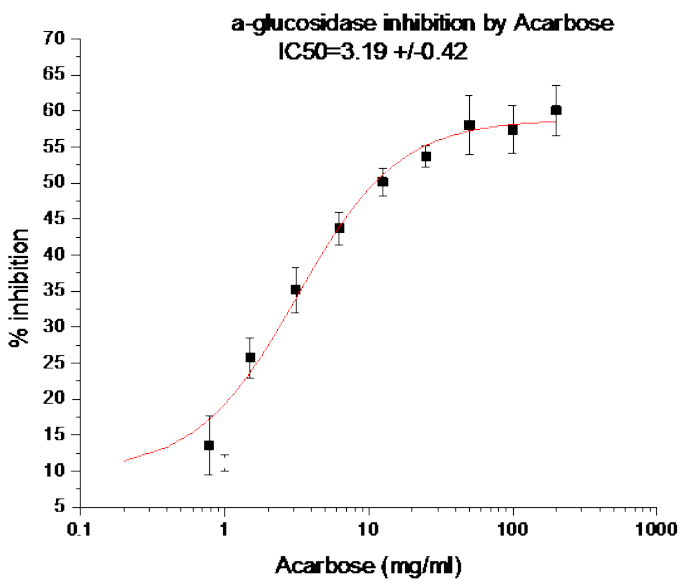

Figure 2. Dose-response curve of Inhibition of alpha-glucosidase from Saccharomyces cerevisiae by acarbose. The results are expressed in mean \pm standard deviation. 


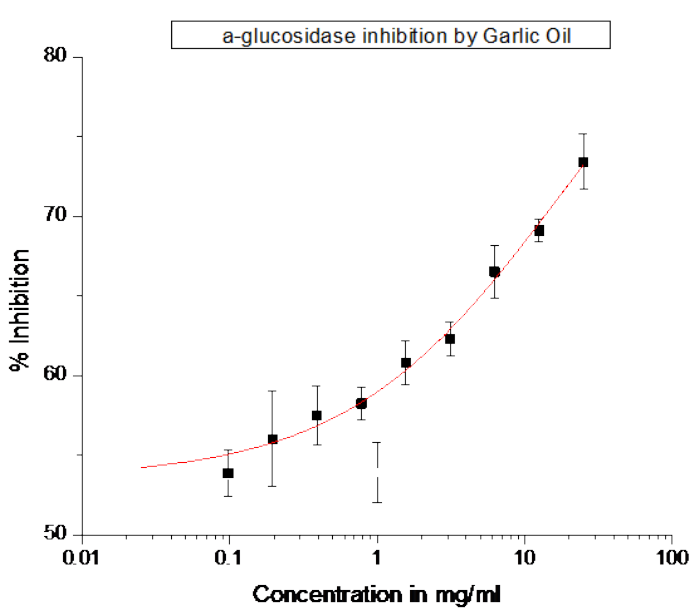

Figure 3. Dose-response curve of Inhibition of alpha-glucosidase from Saccharomyces cerevisiae by garlic (Allium sativum). The results are expressed in mean \pm standard deviation.

\section{Discussion}

The results of this study showed that garlic inhibited alpha-glucosidase from Sachromycetes cerevisiae. Acarbose is in the market and used currently as an antidiabetic agent. It is a carbohydrate analog that acts within the intestine to inhibit alpha-glucosidase, an enzyme necessary for the conversion of complex starches, oligosaccharides, and disaccharides to the monosaccharides that can be transported out of the intestine to the blood stream. As a result of slowed absorption, postprandial hyperglycemia is reduced. It lacks effect on fasting blood sugar. It can be used as monotherapy or in combination with other antidiabetic drugs. Garlic oil or Diallyl trisulfide (DATS) is a garlic-derived organosulfur compound, which has demonstrated many beneficial effects. Behavioral assessment showed that DATS could inhibit $\mathrm{n}$-hexane induced neurotoxicity, demonstrated by the improvement of the grip strength and decline of gait scores [18]. Many animal models have reported of sugar lowering effect of garlic [19] [20] [21] [22]. A study was designed to systematically evaluate the clinical efficacy and safety of garlic supplement in the management of type 2 diabetes mellitus (T2DM). The study confirmed that garlic supplement plays positive and sustained roles in blood glucose, total cholesterol, and high/low density lipoprotein regulation in the management of T2DM [23].

In humans, it has been demonstrated that garlic supplementation with standard antidiabetic agent provides better diabetic control in type 2 diabetes [23]. The beneficial effects of garlic are mainly attributed to the presence of volatile sulfur compounds like alliin, allicin, diallyl disulfide, diallyl trisulfide, diallyl sulfide, S-allyl cysteine, ajoene and allyl mercaptan [12]. Garlic and garlic extracts have also gone through clinical trials have been shown to be effective in reducing insulin resistance [24] [25] [26] [27]. The use of natural products for treatment of diseases has increased over the years. The reasons suggested for the awareness and surge in the use of botanicals include easy availability without 
prescriptions and that natural products or plants may have little or no side effects. Many food products now contain garlic. People use it in many different ways in order to experience health benefits [28]. Many studies have found that garlic has strong antioxidant properties. It has also been found that raw garlic has more antioxidant activity than cooked garlic [29]. Anti-inflammatory and immune protection effects of garlic have also been demonstrated [30].

\section{Conclusion}

We have evaluated the mechanism of action of garlic with respect to alpha-glucosidase inhibition. Garlic has demonstrated a dose-dependent inhibition of alpha-glucosidase in our studies. The rapid increase in diabetes in the world places a huge demand for alternative means of treating diabetes. Due to limitations in many existing conventional antidiabetic dugs, it is crucial to search for alternative remedies for the management of diabetes mellitus. This has led many researchers to investigate traditional remedies as alternatives. Substantial studies have shown that garlic and its bioactive constituents exhibit antioxidant, anti-inflammatory, antibacterial, antifungal, immunomodulatory, cardiovascular protective, anticancer, hepatoprotective, digestive system protective, anti-diabetic, anti-obesity, neuroprotective, and renal protective properties. It is a food substance with many components that are beneficial to man and may have few or no toxicity. It is hoped that further evaluation of different analogs of garlic following purification and characterization will elucidate other benefits and possible mechanisms by which garlic produces an antidiabetic action.

\section{Conflicts of Interest}

The authors declare no conflicts of interest regarding the publication of this paper.

\section{References}

[1] Facts about Diabetes. World Health Organization. https://www.who.int/features/factfiles/diabetes

[2] Statistics about Diabetes. American Diabetes Association. https://www.diabetes.org/resources/statistics/statistics-about-diabetes\#sthash.F8fRk Pqd.dpuf

[3] American Diabetes Association. https://www.diabetes.org/resources/statistics/statistics-about-diabetes\#sthash.F8fRk Pqd.dpuf https://www.who.int/features/factfiles/diabetes/en

[4] Nolte, M.S., Kennedy and Umesh Masharani (2018) Pancreatic Hormones \& Antidiabetic Drugs; Bertram G. Katzung, Basic \& Clinical Pharmacology, 14e.

[5] Choudhury, H., Pandey, M., Hua, K., Mun, C., Jing, J., Kong, L., Liang Yee Ern, L., Ashraf, N., Kit, S., Yee, T., Pichika, M., Gorain, B. and Kesharwania, P. (2018) An Update on Natural Compounds in the Remedy of Diabetes Mellitus: A Systematic Review. Journal of Traditional and Complementary Medicine, 8, 361-376. https://doi.org/10.1016/j.jtcme.2017.08.012 
[6] Harvey, A. (2010) Plant Natural Products in Anti-Diabetic Drug Discovery. Current Organic Chemistry, 14, 1670-1677. https://doi.org/10.2174/138527210792927681

[7] Lebovitz, H.E. (1997) A-Glucosidase Inhibitor. Endocrinology \& Metabolism Clinics of North America, 26, 539-551. https://doi.org/10.1016/S0889-8529(05)70266-8

[8] Fugita, H., Yamagami, T. and Ohshima, K. (2001) Long-Term Ingestion of a Fermented Soybean-Derived Touchi Extract with Alpha-Glucosidase Inhibitory Activity Is Safe and Effective in Humans with Borderline and Mild Type-2 Diabetes. The Journal of Nutrition, 131, 1211. https://doi.org/10.1093/jn/131.8.2105

[9] Ramírez, G., Zavala, M., Pérez, J. and Zamilpa, A. (2012) In Vitro Screening of Medicinal Plants Used in Mexico as Antidiabetics with Glucosidase and Lipase Inhibitory Activities. Evidence-Based Complementary and Alternative Medicine, 2012, Article ID: 701261. https://doi.org/10.1155/2012/701261

[10] Wehmeier, U.F. and Piepersberg, W. (2004) Biotechnology and Molecular Biology of the $\alpha$-Glucosidase Inhibitor Acarbose. Applied Microbiology and Biotechnology, 63, 613-625. https://doi.org/10.1007/s00253-003-1477-2

[11] Ríos, J.L., Francini, F. and Schinella, G.R. (2015) Natural Products for the Treatment of Type 2 Diabetes Mellitus. Planta Medica, 81, 975-994.

https://doi.org/10.1055/s-0035-1546131

[12] Akshatha, V.J., Nalini, M.S., D’Souza, C. and Prakash, H.S. (2014) Streptomycete Endophytes from Anti-Diabetic Medicinal Plants of the Western Ghats Inhibit $\alpha$-Amylase and Promote Glucose Uptake. Letters in Applied Microbiology, 58, 433-439. https://doi.org/10.1111/lam.12209

[13] Melzig, M.F. and Funke, I. (2007) Pflanzliche Alpha-Amylasehemmer eine Möglichkeit zur Phytotherapie bei Diabetes Mellitus Typ II? Wiener Medizinische Wochenschrift, 157, 320-324. https://doi.org/10.1007/s10354-007-0433-7

[14] Rahimzadeh, M., Jahanshahi, S., Moein, S. and Moein, M.R. (2014) Evaluation of Alpha-Amylase Inhibition by Urtica dioica and Juglans regia Extracts. Iranian Journal of Basic Medical Sciences, 17, 465-469.

[15] Bayan, L., Koulivand, P. and Gorji, A. (2014) Garlic: A Review of Potential Therapeutic Effects. Avicenna Journal of Phytomedicine, 4, 1-14.

[16] Padiya, R. and Banerjee, S.K. (2013) Garlic as an Anti-Diabetic Agent: Recent Progress and Patent Reviews. Recent Patents on Food, Nutrition \& Agriculture, 5, 105-127. https://doi.org/10.2174/18761429113059990002

[17] Ramirez, G., Zavala, M., Perez, J. and Zamilpa, A. (2012) In Vitro Screening of Medicinal Plants Used in Mexico as Antidiabetics with Glucosidase and Lipase Inhibitory Activity. Evidence-Based Complementary and Alternative Medicine, 2012, Article ID: 701261. https://doi.org/10.1155/2012/701261

[18] Wang, S., Li, M., Wang, X., Li, X., Yin, H., Jiang, L., Han, W., Irving, G., Zeng, T. and Xie, K. (2017) Diallyl Trisulfide Attenuated n-Hexane Induced Neurotoxicity in Rats by Modulating P450 Enzymes. Chemico-Biological Interactions, 265, 1-7. https://doi.org/10.1016/j.cbi.2017.01.013

[19] Lee, H.S., Lim, W.C., Lee, S.J., Lee, S.H., Lee, J.H. and Cho, H.Y. (2016) Anti-Obesity Effect of Garlic Extract Fermented by Lactobacillus Plantarum bl2 in Diet-Induced Obese Mice. Journal of Medicinal Food, 19, 823-829. https://doi.org/10.1089/jmf.2016.3674

[20] Eidi, A., Eidi, M. and Esmaeili, E. (2005) Antidiabetic Effect of Garlic (Allium sativum L.) in Normal and Streptozotocin-Induced Diabetic Rats. Phytomedicine, 13, 624-629. https://doi.org/10.1016/j.phymed.2005.09.010

[21] Poonam, T., Prakash, G.P. and Kumar, L.V. (2013) Influence of Allium sativum Ex- 
tract on the Hypoglycemic Activity of Glibenclamide: An Approach to Possible Herb-Drug Interaction. Drug Metabolism and Drug Interactions, 28, 225-230. https://doi.org/10.1515/dmdi-2013-0031

[22] Shang, A., Cao, S.Y., Xu, X.Y., Gan, R.Y., Tang, G.Y., Corke, H., Mavumengwana, V. and Li, H.B. (2019) Bioactive Compounds and Biological Functions of Garlic (Allium sativum L.). Foods, 8, pii: E246. https://doi.org/10.3390/foods8070246

[23] Wang, J., Zhang, X., Lan, H.H. and Wang, W. (2017) Effect of Garlic Supplement in the Management of Type 2 Diabetes Mellitus (T2DM): A Meta-Analysis of Randomized Controlled Trials. Food \& Nutrition Research, 61, Article ID: 1377571. https://doi.org/10.1080/16546628.2017.1377571

[24] Ashraf, R., Aamir, K., Shaikh, A.R., et al. (2005) Effects of Garlic on Dyslipidemia in Patients with Type 2 Diabetes Mellitus. Journal of Ayub Medical College Abbottabad, 17, 60-64.

[25] Sobenin, I.A., Nedosugova, L.V., Filatova, L.V., et al. (2008) Metabolic Effects of Time-Released Garlic Powder Tablets in Type 2 Diabetes Mellitus: The Results of Double-Blinded Placebo-Controlled Study. Acta Diabetologica, 45, 1-6. https://doi.org/10.1007/s00592-007-0011-x

[26] Ashraf, R., Khan, R.A. and Ashraf, I. (2011) Effects of Garlic on Blood Glucose Levels and HbAlc in Patients with Type 2 Diabetes Mellitus. Journal of Medicinal Plants Research, 5, 2922-2928.

[27] Atkin, M., Laight, D. and Cummings, M.H. (2016) The Effects of Garlic Extract upon Endothelial Function, Vascular Inflammation, Oxidative Stress and Insulin Resistance in Adults with Type 2 Diabetes at High Cardiovascular Risk. A Pilot Double Blind Randomized Placebo Controlled Trial. Journal of Diabetes and its Complications, 30, 723-727. https://doi.org/10.1016/j.jdiacomp.2016.01.003

[28] Tsai, C.-W., Chen, H.-W., Sheen, L.-Y. and Lii, C.-K. (2012) Garlic: Health Benefits and Actions. BioMedicine, 2, 17-29. https://doi.org/10.1016/j.biomed.2011.12.002

[29] Hayat, S., Cheng, Z., Ahmad, H., Ali, M., Chen, X. and Wang, M. (2016) Garlic from Remedy to Stimulant: Evaluation of Antifungal Potential Reveals Diversity in Phytoalexin Allicin Content among Garlic Cultivars; Allicin Containing Aqueous Garlic Extracts Trigger Antioxidants. Frontiers in Plant Science, 7, 1235. https://doi.org/10.3389/fpls.2016.01235

[30] Percival, S.S. (2016) Aged Garlic Extract Modifies Human Immunity. The Journal of Nutrition, 146, 433S-436S. https://doi.org/10.3945/jn.115.210427 